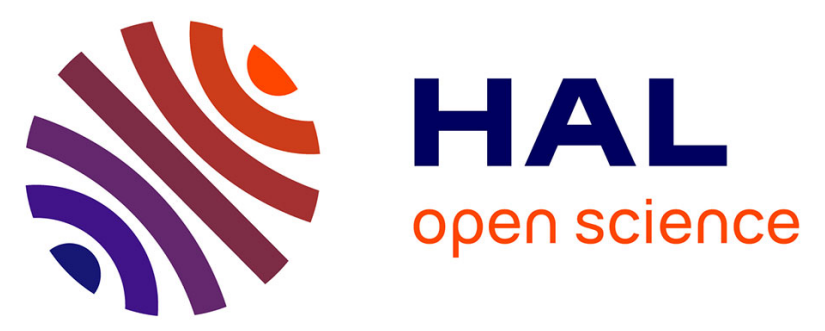

\title{
MMSE turbo equalization using a low complexity series expansion to approximate the matrix inversion in frequency selective MIMO channels
}

\author{
Nicolas Le Josse, Christophe Laot, Karine Amis Cavalec
}

\section{To cite this version:}

Nicolas Le Josse, Christophe Laot, Karine Amis Cavalec. MMSE turbo equalization using a low complexity series expansion to approximate the matrix inversion in frequency selective MIMO channels. 5th international symposium on Turbo Codes \& related topics, 1-5 septembre, Lausanne, Suisse, Sep 2008, Lausanne, Switzerland. hal-02120751

\section{HAL Id: hal-02120751 \\ https://hal.science/hal-02120751}

Submitted on 11 Jun 2021

HAL is a multi-disciplinary open access archive for the deposit and dissemination of scientific research documents, whether they are published or not. The documents may come from teaching and research institutions in France or abroad, or from public or private research centers.
L'archive ouverte pluridisciplinaire HAL, est destinée au dépôt et à la diffusion de documents scientifiques de niveau recherche, publiés ou non, émanant des établissements d'enseignement et de recherche français ou étrangers, des laboratoires publics ou privés. 


\title{
MMSE turbo equalization using a low complexity series expansion to approximate the matrix inversion in frequency selective MIMO channels
}

\author{
Nicolas Le Josse, Christophe Laot and Karine Amis \\ Institut TELECOM; TELECOM Bretagne; Signal and Communications Dept. \\ Technopôle Brest-Iroise, CS 83818, 29238 Brest Cedex 3, France \\ Email:\{nicolas.lejosse;christophe.laot;karine.amis\}@ telecom-bretagne.eu \\ Tel : 33 (0)2 290010 14, Fax: 33 (0)2 29001012
}

\begin{abstract}
The paper describes a novel approach to overcome the need for matrix inversion required by Minimum Mean Square Error (MMSE) turbo equalization in MIMO systems. In particular, turbo MMSE equalization based on an efficient series expansion from a truncated Taylor series expansion to approximate the matrix inversion is addressed. By adjusting a scaling factor, the proposed series expansion is directly optimized according to a fixed order with respect to a system performance criterion for each source to be estimated. The proposed approach enables low complexity receiver without the need for a complicated eigenvalue calculation procedure. From the first order, the proposed series expansion ensures the best bit error rate performance with a simple non iterative receiver in comparison with previous approaches. Taking advantage of the iterative process, the resulting MMSE turbo equalizer reaches the matched filter bound.
\end{abstract}

Index Terms-Low complexity receiver, matrix inversion, MIMO systems, MMSE turbo equalization, series expansion.

\section{INTRODUCTION}

The transmission of information for most communication channels including multiple-input multiple-output (MIMO) systems and multipath fading is subject to intersymbol interference. MMSE turbo equalization [1]-[5] has been proven effective for removing intersymbol interference. It consists of a minimum mean square error (MMSE) equalizer and a decoder. When combined with space time bit interleaved coded modulation (ST-BICM) systems [6], [7], MMSE turbo equalization achieves optimal performance by taking fully advantage of the available diversity [8]-[13]. Unfortunately, its computational cost is too high and makes its implementation difficult since it requires large matrix inversions depending on the size of the system [14], [15].

To overcome the need for matrix inversion the authors proposed in [16]-[19] an approximate MMSE equalizer employing a finite sum of weighted matrix polynomials that results from the Cayley-Hamilton theorem [20]. The coefficients are chosen to optimize some performance measure at the equalizer output. It has been extended and applied to MMSE turbo equalization in the context of multiuser detection in [21]. In particular, by taking advantage of the iterative process, the authors show that performance loss due to the polynomial approximation is negligible after few iterations.
However, the complexity involved in the weight optimization problem does not seem to be easier than performing an exact matrix inversion [17], [22].

Alternatively, an approximate MMSE equalization based on an optimal series expansion from a truncated Taylor series expansion is derived in [23]. By adjusting a scaling factor, the series expansion is directly optimized according to a fixed order with respect to a system performance criterion. In comparison with previous approaches [24], [25], the proposed series expansion ensures the best performance at the equalizer output in addition to low complexity which mainly depends on the computation of only one scaling factor as a function of the equalizer coefficients. This provides a much greater potential complexity reduction in comparison with a complicated eigenvalue calculation procedure.

In this paper, the approach proposed in [23] is expanded to derive an efficient MMSE turbo equalizer based on the first order series expansion to approximate the matrix inversion in a MIMO context. Compared to previous approaches, the proposed method improves the bit error rate (BER) performance for the first iterations. By taking advantage of the iterative process, the resulting MMSE turbo equalizer exhibits sufficient performance to reach the matched filter bound with approximately the same convergence speed to that of the corresponding MMSE turbo equalizer with exact matrix inversion.

In section II, the MIMO transmission scheme is described. In section III, the proposed approximate MMSE turbo equalizer equation based on series expansion is derived. In sections $\mathrm{V}$, simulation results show the receiver efficiency in comparison with that of the corresponding MMSE turbo equalizer with exact matrix inversion.

\section{MIMO SYSTEM DESCRIPTION}

We consider a single carrier transmission in MIMO STBICM system made of $n_{T}$ transmit and $n_{R}$ receive antennas over a frequency selective channel. As illustrated in Fig. 1, the information bit stream is encoded and then bit-interleaved

This work was supported in part by France Telecom Research and Development division. 
$\left(\Pi_{b}\right)$. The interleaved streams are then modulated and serial to parallel converted. The transmitted symbols are organized as blocks of $N$ transmitted symbols on each antenna. Consecutive blocks of $N$ transmitted symbols are separated by a guard interval (GI), which prevents from inter-block interference. Each guard interval is assumed to contain $L-1$ zeros (ZeroPadding). We assume a coherent symbol-spaced receiver with perfect carrier and time synchronization such that $y_{n}(d)$, the $d$ th sample on the receive antenna $n$, can be represented by a discrete-time baseband model having $s_{m}(d)$, the $d$ th transmitted symbol from the $m$ th transmit antenna, as input. The transmitted symbols are assumed to be independent, identically distributed (i.i.d) and of variance $\sigma_{s}^{2}$. The complex additive Gaussian noise samples on the receive antenna $n$ of variance $\sigma_{b}^{2}$ are denoted $b_{n}(d)$. For the equalizer derivation, we consider a sliding window of length $N_{F}$ from each antenna at the receiver. By grouping the received samples from all $n_{R}$ received antennas over a block of $N_{F}$ symbol periods, we thus obtain $N$ vectors $\mathbf{y}(d)$ of size $N_{F} n_{R} \times 1$ whose expression is

$$
\underline{\mathbf{y}}(d)=\underline{\mathbf{H} \mathbf{s}}(d)+\underline{\mathbf{b}}(d) \quad d=0, . ., N-1
$$

where $\underline{\mathbf{y}}(d)=\left[\left\{y_{n}(d+k)\right\}_{1 \leq n \leq n_{R}}\right]_{0 \leq k \leq N_{F}-1}$ of dimension $N_{F} n_{R} \times 1, \underline{\mathbf{s}}(d)=\left[\left\{s_{m}(d+k)\right\}_{1 \leq m \leq n_{T}}\right]_{N_{F_{1}} \leq k \leq N_{F_{2}}+L-1}$ of dimension $\left(N_{F}+L-1\right) n_{T} \times 1$ and $\underline{\mathbf{b}}(d)=\left[\left\{b_{n}(d+\right.\right.$ $\left.k)\}_{1 \leq n \leq n_{T}}\right]_{1<k<N_{F}-1}$ of dimension $N_{F} n_{R} \times 1$. $\underline{\mathbf{H}}$ represents the $N_{F} n_{R} \times\left(N_{F}+L-1\right) n_{T}$ block-Toeplitz channel matrix

$\underline{\mathbf{H}}=\left[\begin{array}{ccccc}\mathbf{H}(0) & \ldots & \mathbf{H}(L-1) & 0 & \ldots \\ 0 & \mathbf{H}(0) & \ldots & \mathbf{H}(L-1) & 0 \\ \vdots & \ddots & \ddots & \ddots & \vdots \\ 0 & \ldots & \mathbf{H}(0) & \ldots & \mathbf{H}(L-1)\end{array}\right]$

consisting of blocks $\mathbf{H}(l)=\left[h_{n, m}(l)_{\substack{1 \leq m \leq n_{T} \\ 1 \leq n \leq n_{R}}}\right]_{0 \leq l \leq L-1}$ of dimension $n_{R} \times n_{T}$ where the discrete linear filter $h_{n, m}(l)_{l=0, . ., L-1}$ of length $L$ denotes the discrete-time equivalent channel model between transmit antenna $m$ and receive antenna $n$.

\section{EFFICIENT APPROXIMATE MMSE TURBO EQUALIZER BASED ON SERIES EXPANSION}

The structure of the investigated iterative receiver is shown in Fig. 1. The receiver consists of the concatenation of a Soft Input / Soft Output equalizer and a Soft Input / Soft Output decoder exchanging information on the coded bits in an iterative manner according to the MMSE turbo equalization principle [3]-[5]. This section focuses on the derivation of the proposed MMSE turbo equalizer based on series expansion.

\section{A. MMSE turbo equalizer derivation}

When considering turbo equalization, the decoder should provide the equalizer with extrinsic information for the encoded bits. Interestingly, we have noticed performance improvements for MMSE turbo equalization when feeding back the entire a posteriori information. The same obervation has been reported in [26], [27]. Let us denote $\tilde{s}_{m}(d)$ the soft estimate of transmitted symbol $s_{m}(d)$ computed from the $a$ posteriori probabilities provided by the decoder. As in [4],
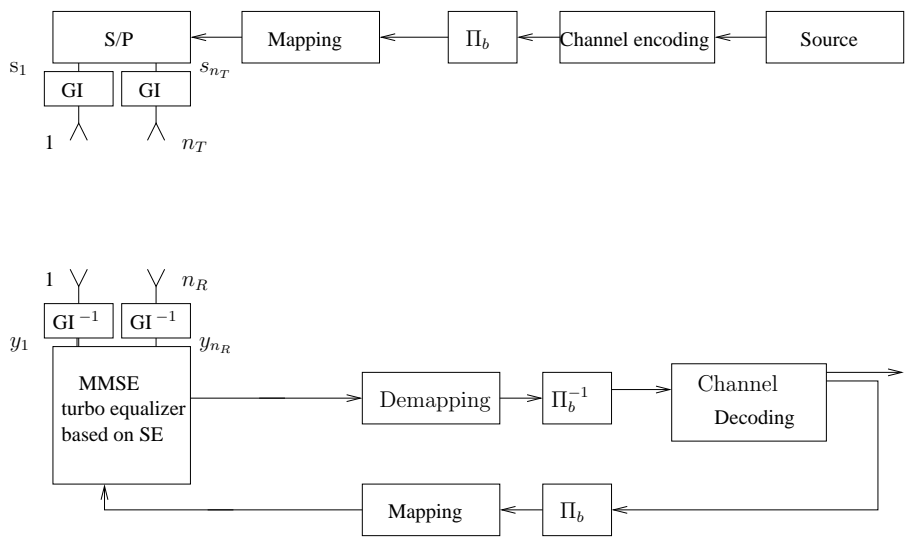

Fig. 1. Single carrier transmission in ST-BICM systems with MMSE turbo equalization based on efficient series expansion (SE) at the receiver.

[26], the soft symbols $\left\{\tilde{s}_{m}(d)\right\}_{m=1, \ldots, n_{T}}^{d=0, \ldots-1}$ are of variance $\sigma_{\tilde{s}}^{2}$ approximated by $\sigma_{\tilde{s}}^{2}=\frac{1}{N n_{T}} \sum_{m=1}^{n_{T}} \sum_{d=0}^{N-1}\left|\tilde{s}_{m}(d)\right|^{2}$ where a time average over a block of $N$ symbols from all $n_{R}$ received antenna is used. The resulting MMSE turbo equalizer coefficients remain invariant over a block of $N$ transmitted symbols. Using these data estimates, and the channel coefficients, a soft replica of the multi-antenna interference (MAI) and intersymbol interference (ISI) is computed for each of the $n_{T}$ antennas and then substracted from the total received signal

$$
\underline{\mathbf{y}}(d)=\underline{\mathbf{y}}(d)-\underline{\mathbf{H} \tilde{\mathbf{s}}}(d)
$$

where $\underline{\tilde{\mathbf{s}}}(d)=\left[\left\{\tilde{s}_{m}(d+k)\right\}_{1 \leq m \leq n_{T}}\right]_{N_{F_{1}} \leq k \leq N_{F_{2}}+L-1}$ of dimension $n_{T}\left(N_{F}+L-1\right) \times 1$. MMSE turbo equalization consists in cancelling soft ISI and MAI, and filtering based on minimum mean square error (MMSE) criteria

$$
\mathrm{E}\left[\left|\underline{\mathbf{w}}_{m}^{\mathrm{H}}\left(\underline{\tilde{\mathbf{y}}}^{(d)}+\underline{\mathbf{h}}_{\Delta_{m}} \tilde{s}_{m}(d)\right)-s_{m}(d)\right|^{2} \mid \underline{\tilde{\mathbf{s}}}(d)\right], m=1, . ., n_{T}
$$

where $\underline{\mathbf{h}}_{\Delta_{m}}$ is the $\Delta_{m}^{t h}$ column vector of channel matrix $\underline{\mathbf{H}}$ corresponding to the different fadings experienced by the desired component. The MMSE filter input signal is independent of the soft estimate $\tilde{s}_{m}(d)$ according to the turbo principle. More explicitly, the equalizer consists of a $N_{F} n_{R} \times 1$ dimensional filter $\underline{\mathbf{w}}_{m}$ which minimizes the cost function (4) such that

$$
\underline{\mathbf{w}}_{m}^{\mathrm{H}}=\alpha_{m} \underline{\mathbf{h}}_{\Delta_{m}}^{\mathrm{H}} \underline{\boldsymbol{\Sigma}}^{-1}
$$

in which $\underline{\boldsymbol{\Sigma}}$ is the $n_{R} N_{F} \times n_{R} N_{F}$ covariance matrix of the residual interference given by $\underline{\boldsymbol{\Sigma}}=\sigma_{s}^{2} \underline{\mathbf{H H}}^{\mathrm{H}}(1-r)+$ $\sigma_{b}^{2} \mathrm{I}_{N_{F} n_{R} \times N_{F} n_{R}}$ where we define $r=\frac{\sigma_{s}^{2}}{\sigma_{s}^{2}}$ as the degree of a priori information reliability. It can be seen that $r=1$ (resp. $r=0$ ), corresponds to perfect (resp. unavailable) $a$ priori information from the decoder output. The index $\Delta_{m}$ is the decision delay parameter required for the derivation of the equalizer transfer function such that $\Delta_{m}=N_{F_{1}}+$ 1. The intermediate variable $\alpha_{m}$ is computed as $\alpha_{m}=$ $\frac{\sigma_{s}^{2}}{1+r \sigma_{s}^{2} \underline{\underline{H}}_{\Delta m}^{\mathrm{H}} \underline{\boldsymbol{\Sigma}}^{-1} \underline{\mathbf{h}}_{\Delta_{m}}}$. Turbo equalization requires a minimum filter length $\bar{N}_{F}$ to achieve good performance [28] at the expense of high computational complexity mainly due to the calculation of $\underline{\boldsymbol{\Sigma}}^{-1}$ of dimension $\left(N_{F} n_{R} \times N_{F} n_{R}\right)$. 


\section{B. Previous approaches}

Let $\lambda_{i}, i=1, . ., N_{F} n_{R}$ denote the positive real eigenvalues of $\underline{\boldsymbol{\Sigma}}$. Now consider a scaling factor $\psi \in \mathbb{R}$, which is determined for the matrix $\underline{\Sigma}$ such that

$$
\left|1-\psi \lambda_{i}\right|<1, \quad i=1, . ., N_{F} n_{R}
$$

If the previous condition is satisfied, we can introduce the series expansion

$$
\underline{\boldsymbol{\Sigma}}^{-1}=\psi \sum_{i=0}^{\infty}\left(\mathbf{I}_{N_{F} n_{R}}-\psi \underline{\boldsymbol{\Sigma}}\right)^{i}
$$

By considering the $K$ th order series expansion in $\underline{\boldsymbol{\Sigma}}$ to approximate the matrix inversion in (5), the corresponding equalizer is given by

$$
\begin{gathered}
\underline{\mathbf{w}}_{m(K)}^{\mathrm{H}}=\alpha_{m(K)} \psi \underline{\mathbf{h}}_{\Delta_{m}}^{\mathrm{H}}\left(\mathbf{I}_{N_{F} n_{R}}+\left(\mathbf{I}_{N_{F} n_{R}}-\psi \underline{\boldsymbol{\Sigma}}\right)+\right. \\
\left.\left(\mathbf{I}_{N_{F} n_{R}}-\psi \underline{\boldsymbol{\Sigma}}\right)^{2}+\ldots+\left(\mathbf{I}_{N_{F} n_{R}}-\psi \underline{\boldsymbol{\Sigma}}\right)^{K}\right)
\end{gathered}
$$

which converges from the matched-filter $(K=0)$ detector to the MMSE equalizer $(K \rightarrow \infty)$, as the order $K$ grows from zero to infinity, i.e. $\lim _{K \rightarrow \infty} \underline{\mathbf{w}}_{m(K)}^{\mathrm{H}}=\underline{\mathbf{w}}_{m}^{\mathrm{H}}$. From this point of view, it is advantageous for computational simplicity to keep $K$ as low as possible while still maintaining sufficient performance. Let $\lambda_{\min }$ and $\lambda_{\max }$ denote the smallest and the largest eigenvalues of $\underline{\boldsymbol{\Sigma}}$. A simple analysis shows that the convergence of $\underline{\mathbf{w}}_{m(K)}^{\mathrm{H}}$ to $\underline{\mathbf{w}}_{m}^{\mathrm{H}}$ as $K \rightarrow \infty$ is ensured by choosing $\psi$ in the range of $0<\psi<\frac{2}{\lambda_{\max }}$. In [24] [29], the authors propose to set

$$
\psi=\frac{2}{\operatorname{trace}(\underline{\boldsymbol{\Sigma}})}
$$

since trace $(\underline{\boldsymbol{\Sigma}}) \geq \lambda_{\max }$. There is a possibility of estimating a more suitable scaling factor if the eigenvalues of $\underline{\Sigma}$ are known. The fastest convergence rate takes place when the two extreme modes $\left|1-\psi \lambda_{\min }\right|$ and $\left|1-\psi \lambda_{\max }\right|$ are identical such that

$$
\psi=\frac{2}{\lambda_{\min }+\lambda_{\max }}
$$

These results are well-known and have previously been presented [25]. It should be noted that when the simulation context is associated with a large spread of eigenvalues, high order $K$ may be required to keep high performance, at the expense of computational complexity.

\section{Proposed approach}

Instead of ensuring that $\underline{\mathbf{w}}_{m(K)}^{\mathrm{H}}$ performs the fastest convergence rate to $\underline{\mathbf{w}}_{m}^{\mathrm{H}}$ as $K$ grows from zero to infinity, the proposed approach [23] consists in setting $\psi$ such that $\underline{\mathbf{w}}_{m(K)}^{\mathrm{H}}$ achieves an optimum for some performance measure according to a fixed order $K$, for each source $m=1, . ., n_{T}$ to be estimated. In that sense, we denote $\psi_{m}(K)$ the new scaling factor. We propose to derive an efficient approximate MMSE turbo equalizer based on the first order series expansion of $\underline{\boldsymbol{\Sigma}}^{-1}$ that achieves the best signal interference to noise ratio (SINR) at the equalizer output. More explicitly, the first order approximate MMSE equalizer equation is given by

$$
\underline{\mathbf{w}}_{m(1)}^{\mathrm{H}}=\alpha_{m(1)} \psi_{m}(1) \underline{\mathbf{h}}_{\Delta_{m}}^{\mathrm{H}}\left(\mathbf{I}_{N_{F} n_{R}}+\left(\mathbf{I}_{N_{F} n_{R}}-\psi_{m}(1) \underline{\boldsymbol{\Sigma}}\right)\right)
$$

By considering the expression of the SINR at the equalizer output, the optimum scaling factor $\psi_{m}(1)$ can then be found by solving $\left(\psi_{m}^{\mathrm{opt}}(1)\right)=\underset{\psi_{m}(1)}{\operatorname{argmax}}\left(\frac{\left\|\underline{\mathbf{w}}_{m(1)}^{\mathrm{H}} \underline{\mathbf{h}}_{\Delta_{m}}\right\|^{2}}{\underline{\mathbf{w}}_{m(1)}^{\mathrm{I}} \underline{\mathbf{w}}_{m(1)}}\right)$ under the constraint that $\psi_{m}(1)$ ensures the convergence of $\underline{\mathbf{w}}_{m(K)}^{\mathrm{H}}$ to $\underline{\mathbf{w}}_{m}^{\mathrm{H}}$ as $K \rightarrow \infty$. The optimum scaling factor $\psi_{m}(1)$ that achieves the best SINR at the equalizer output for each source $m=1, . ., n_{T}$ to be estimated, can be expressed as a function of the equalizer coefficients

$$
\psi_{m}(1)=\frac{2}{\frac{\underline{\mathbf{v}}_{m}^{\mathrm{H}} \underline{\boldsymbol{\Theta}}_{m} \underline{\boldsymbol{\Sigma}}_{m}}{\underline{\mathbf{v}}_{m}^{\mathrm{H}} \underline{\boldsymbol{\Theta}}_{m} \underline{\mathbf{v}}_{m}}}
$$

where we define $\underline{\mathbf{v}}_{m}^{\mathrm{H}}=\mathbf{h}_{\Delta_{m}}^{\mathrm{H}} \underline{\boldsymbol{\Sigma}}$ and $\underline{\boldsymbol{\Theta}}_{m}=\left\|\underline{\mathbf{h}}_{\Delta_{m}}\right\|^{2} \mathbf{I}_{N_{F} n_{R}}-$ $\underline{\mathbf{h}}_{\Delta_{m}} \underline{\mathbf{h}}_{\Delta_{m}}^{\mathrm{H}}$ [23]. It can be shown that the resulting $\psi_{m}(1)$ satisfies the condition (6) if and only if $r<1$. For the case in which $r \rightarrow 1$, a priori information from the decoder output is good enough so that that a matched filter approximation of the proposed MMSE turbo equalizer can efficiently reach the matched filter bound [14]. It is thus proposed that a matched filter is performed at the receiver when $r$ is superior to a given treshold value 0.95 to ensure the convergence of $\underline{\mathbf{w}}_{m(K)}^{\mathrm{H}}$ to $\underline{\mathbf{w}}_{m}^{\mathrm{H}}$ as $K \rightarrow \infty$ and $r \rightarrow 1$. In comparison with previous approaches [24], [25], the proposed series expansion achieves the best SINR at the equalizer output according to a fixed order for each source to be estimated without the need for a complicated eigenvalue calculation procedure.

\section{Simulated SyStem PARAMETERS}

To evaluate the performance of the proposed approximate MMSE turbo equalizer based on series expansion, we consider a block transmission MIMO system with $n_{T}=n_{R}=2$ and perfect channel state information at the receiver. The frame size is 512 information bits. Information bits are first encoded with a convolutional code of rate $1 / 2$ and octal code generator polynomials $(5,7)$. The coded bits are then interleaved by a pseudo-random permutation, serial to parallel converted and 4PSK modulated. The transmitted symbols from each antenna have an equal variance of $\sigma_{s}^{2}=P_{s} / n_{T}$ where $P_{s}$ denotes the total transmitted average power. We assume that channel impulse responses between $n_{T}$ transmit and $n_{R}$ receive antennas are uncorrelated and consist of $L=10$ paths separated by a symbol duration and of equal average power $1 / L$. The coefficients $h_{n, m}(l)_{l=0, . ., L-1}$ are assumed to be complex Rayleigh-distributed, i.i.d, of zero mean and satisfying the power normalization constraint $\mathrm{E}\left[\sum_{l=0}^{L-1}\left|h_{n, m}(l)\right|^{2}\right]=1$. In the MMSE equalizer, the filter coefficient number is set to $\left(N_{F}=21, N_{F_{1}}=N_{F_{2}}=10\right)$. Simulations have shown that setting the filter coefficient number $N_{F}=21$ and the decision delay parameter $\Delta=10$ is sufficient to achieve good BER performance [28].

\section{Simulation RESUlts AND DiscUSSION}

In the following, TE MMSE-SEopt $(K)$ (resp. TE MMSESEev $(K)$ ) denotes the $K$ th order approximate MMSE turbo equalizer computed from (12) (resp. (10)). To compare the interest of the proposed method with that in [24], the $K$ th order approximate MMSE equalizer performance obtained from (9) 


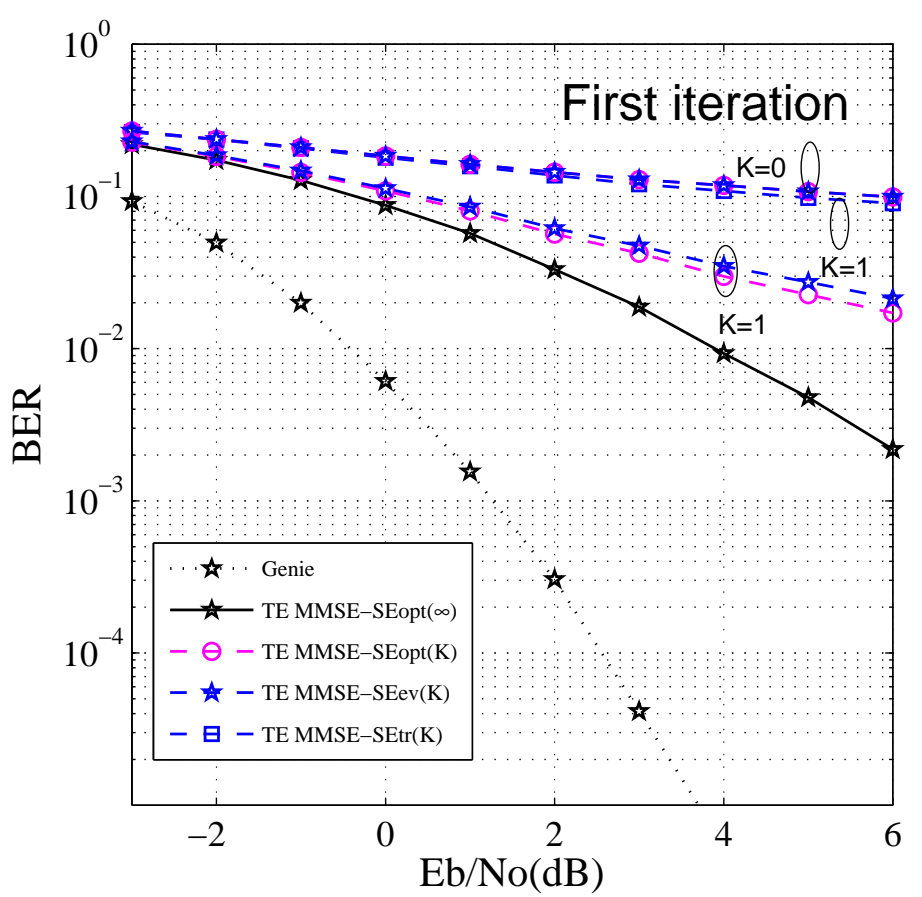

Fig. 2. BER performance after the first decoding iteration of the proposed $K$ th order approximate MMSE equalizer (MMSE-SEopt $(K))$ for $K=0,1$ and the corresponding MMSE equalizer with exact matrix inversion (MMSESEopt $(\infty))$ as a function of $\frac{E_{b}}{N_{0}}-2 \mathrm{Tx} 2 \mathrm{Rx}-\mathrm{CC}(5,7)-4-\mathrm{PSK}-\mathrm{L}=10$.

is also reported (TE MMSE-SEtr( $K)$ ). In Fig. 2 and Fig. 3, the BER after the first and the sixth decoding iterations is plotted as a function of $E_{b} / N_{0}$ for $K=0,1$ and $K \rightarrow \infty$. The performance of the TE MMSE-SEopt $(\infty)$ with perfect a priori information from the decoder is also reported as a reference. The so-called genie MMSE turbo equalizer suppresses MAI and ISI from each transmit antenna, from the received signal.

\section{A. Non iterative receiver performance (first iteration)}

In Fig. 2, simulation results after one iteration i.e without a priori information stand for performance with a simple non iterative receiver. Interestingly, the TE MMSE-SEopt(1) performance enables a good compromise between the matched filter $(K=0)$ and the MMSE equalizer $(K \rightarrow \infty)$ with exact matrix inversion, i.e. complexity and ISI+MAI reduction. For a BER of $1.10^{-1}$, the MMSE-SEopt(1) performs better (resp. worse) by about $5.5 \mathrm{~dB}$ (resp. $0.5 \mathrm{~dB}$ ) than the MMSE-SEopt(0) (resp. the MMSE-SEopt $(\infty)$ ). Compared to the TE MMSESEev(1) BER, that of the TE MMSE-SEopt(1) is improved by $0.2 \mathrm{~dB}$. The TE MMSE-SEopt(1) equalizer is able to retrieve a large part of the ISI and MAI from the received signal and high performance can already be obtained.

At $\frac{E_{b}}{N_{0}}>2 \mathrm{~dB}$, we observe that the performance gap between the MMSE-SEopt $(K)$ for $K=0,1$ and the MMSE-SEopt $(\infty)$ increases. For a BER of $2.10^{-2}$, the TE MMSE-SEopt(1) performs worse by $2.5 \mathrm{~dB}$ from the TE MMSE-SEopt $(\infty)$. The ISI phenomenon dominates over the noise such that the equalizer has to invert the channel impulse response. As a consequence, a high order series expansion is necessary to keep high performance. Compared to the TE MMSE-SEopt(1) BER, the TE MMSE-SEtr(1) BER, leads to a significant performance loss.

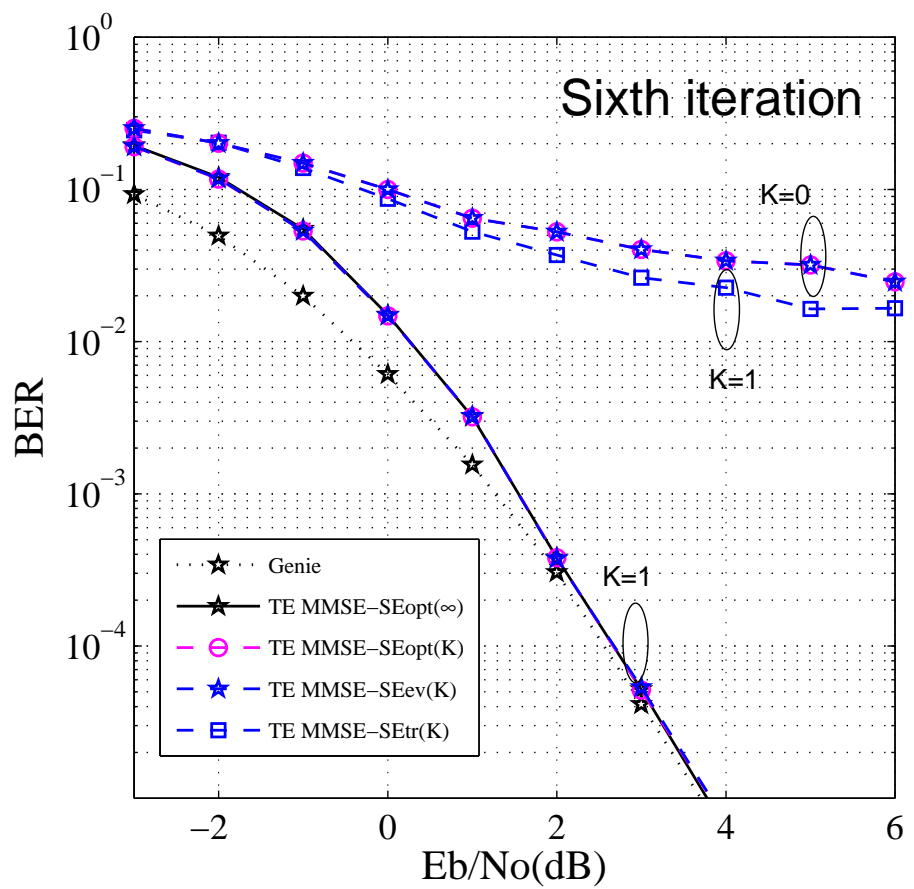

Fig. 3. BER performance after the sixth decoding iteration of the proposed $K$ th order approximate MMSE equalizer (MMSE-SEopt $(K))$ for $K=0,1$ and the corresponding MMSE equalizer with exact matrix inversion (MMSESEopt $(\infty))$ as a function of $\frac{E_{b}}{N_{0}}-2 \mathrm{Tx} 2 \mathrm{Rx}-\mathrm{CC}(5,7)-4-\mathrm{PSK}-\mathrm{L}=10$.

\section{B. Iterative receiver performance (sixth iteration)}

The Fig. 3 shows that the sixth decoding iteration corresponding to the TE MMSE-SEopt $(K)$ (resp. TE MMSE$\operatorname{SEev}(K))$ for $K=1$ is very close to that of the genie decoder. On the other hand, taking $K=0$ brings a noticeable loss degradation. For $K=0$, the BER at the TE MMSE$\operatorname{SEopt}(0)$ equalizer output is exactly the same as the one available at the TE MMSE-SEev(0) (resp. TE MMSE-SEtr(0)) output (matched filter). For a BER of $2 \cdot 10^{-2}$ at the decoder output, the TE MMSE-SEopt(0) (resp. TE MMSE-SEev(0), TE MMSE-SEtr(0)) performs worse by $5.5 \mathrm{~dB}$ compared to the genie decoder BER. Thus, the TE MMSE-SEopt(1) enables a good compromise between the matched filter $(K=0)$ and the MMSE equalizer $(K \rightarrow \infty)$ at the first iteration to reach the matched filter bound after a few iterations. Comparing the TE MMSE-SEopt(1) to the TE MMSE-SEev(1), the TE MMSESEopt(1) exhibits similar performance with that of the TE MMSE-SEev(1) without the need for a complicated eigenvalue calculation procedure as in (10).

\section{Iterative receiver convergence}

In Fig. 4, a further evaluation is performed to compare the iterative receiver convergence of the $K$ th order approximate MMSE turbo equalizer TE MMSE-SEopt $(K)$ (resp. TE $\operatorname{MMSE}-\operatorname{SEev}(K))$ with that of the MMSE equalizer $(K \rightarrow$ $\infty)$. For $\frac{E_{b}}{N_{0}}=3 \mathrm{~dB}$, the BER performance is plotted as a function of the iterations. It can be seen that approximately 4 iterations are required for the MMSE-SEopt(1) (resp. TE MMSE-SEev(1)) and the TE MMSE-SEopt $(\infty)$ to reduce the performance gap by $0.2 \mathrm{~dB}$ compared to the genie decoder output. The improvement brought by the fifth and sixth iterations is negligible. On the other hand, no performance 


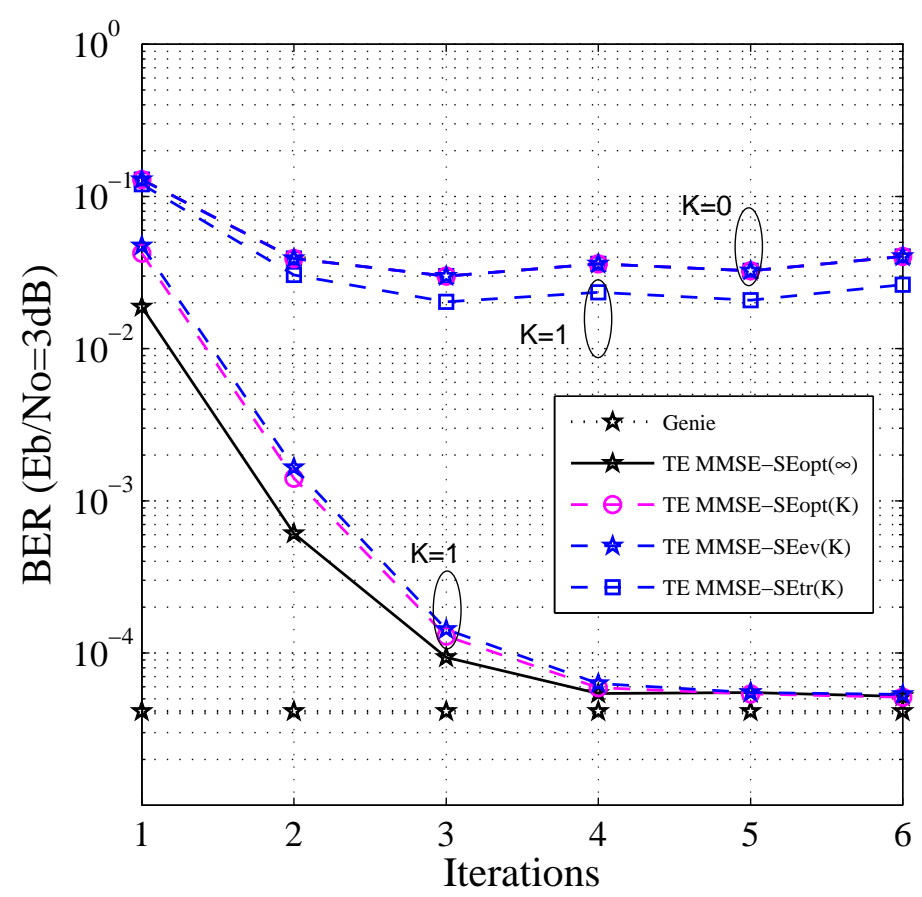

Fig. 4. BER performance of the proposed $K$ th order approximate MMSE equalizer (MMSE-SEopt $(K)$ ) for $K=0,1$ and the corresponding MMSE equalizer with exact matrix inversion (MMSE-SEopt $(\infty)$ ) as a function of the iterations for $\frac{E_{b}}{N_{0}}=3 \mathrm{~dB}-2 \mathrm{Tx} 2 \mathrm{Rx}-\mathrm{CC}(5,7)-4-\mathrm{PSK}-\mathrm{L}=10$.

gain with additional iterations is observed at the TE MMSESEtr(1) output. This demonstrates the efficiency of the MMSESEopt(1) to exploit interference.

\section{CONCLUSION}

This paper describes an innovative and low complexity approach based on series expansion to overcome the need for the complicated matrix inversion required in MMSE turbo equalization derivation. According to the first order, the proposed series expansion TE MMSE-SEopt $(K)$ ensures the best performance with a simple non iterative receiver in comparison with previous approaches (TE MMSE-SEev $(K)$, TE MMSE$\mathrm{SEtr}(K))$. Taking advantage of the iterative process, the resulting MMSE turbo equalizer reaches the matched filter bound with approximately the same convergence speed to that of the corresponding MMSE turbo equalizer with exact matrix inversion. Further to these promising results, important issues should be studied including the computational cost reduction of $\psi_{m}(K)$ derivation and making the proposed approach more widely applicable.

\section{REFERENCES}

[1] C. Douillard, A. Picart, P. Didier, M. Jézéquel, C. Berrou, and A. Glavieux, "Iterative Correction of Intersymbol Interference: Turboequalization," European Trans. on Telecommunications, vol. 6, no. 5, pp.507-511, Sept. 1995.

[2] G. Bauch and V. Franz, "A comparison of soft-in/soft-out algorithms for Turbo detection," Proc. Intern. Conf. on Telecomm., (ICT 1998), pp. 259-263, June 1998.

[3] A. Glavieux, C. Laot, and J. Labat, "Turbo equalization over a frequency selective channel," Proc. Int. Symp. Turbo Codes, pp.96-102, Sept. 1997.

[4] M. Tüchler, A. Singer, and R. Koetter, "Minimum mean squared error equalization using a priori information," IEEE Trans. Signal processing, vol. 50, no. 3, pp.673-683, March 2002.

[5] X. Wang and H. Poor, "Iterative (turbo) soft interference cancellation and decoding for coded CDMA," IEEE Trans. Commun., vol. 47, no. 7, pp.1046-1061, July 1999.
[6] G. Caire, G. Taricco, and E. Biglieri, "Bit Interleaved Coded Modulation," IEEE Trans. Information Theory, vol. 44, no. 3, pp.927-946, May 1998.

[7] J. Boutros, F. Boixadera, and C. Lamy, "Bit-Interleaved Coded Modulation for Multiple-input Multiple-output Channels," IEEE Int. Symp. Spread Spectrum Techniques and Appl., Parsippany, New Jersey, USA, pp. 123-126, Sept. 2000.

[8] A. Tonello, "On Turbo Equalization of Interleaved Space-Time Codes," IEEE Veh. Technol. Conf. (VTC 2001), vol. 2, pp. 887-891, Oct. 2001.

[9] S. Ariyavisitakul, "Turbo Space-Time Processing to Improve Wireless Channel Capacity," IEEE Trans. Commun., vol. 48, no.8, pp. 1347-1358, Aug. 2000.

[10] S. Chtourou, R. Visoz, and A. O. Berthet, "A Class of Low Complexity Iterative Equalizers for Space -Time BICM over MIMO Block Fading Multipath AWGN Channel," Comm. 2004, IEEE International Conf., vol. 1, pp. 618-624, June 2004.

[11] X. Wautelet, A. Dejonghe, and L. Vandendorpe, "MMSE-Based Turbo Receiver for Space-Time BICM Over Frequency-Selective MIMO Fading Channels," IEEE Trans. Signal Processing, vol. 52, no. 6, pp.18041809, June 2004.

[12] N. Le Josse, C. Laot, and K. Amis, "Performance Validation for MMSE Turbo Equalization in ST-BICM Systems," IEEE Veh. Technol. Conf. (VTC 2006), pp. 1-5, Sept. 2006.

[13] T. Ait Idir, S. Saoudi, and N. Naja, "Space Time Turbo Equalization with Successive Interference cancellation for frequency selective MIMO Channels," IEEE Trans. Veh. Technol., vol. 57, no. 4, July 2008.

[14] H. Oomori, T. Asai, and T. Matsumoto, "A matched filter approximation for SC/MMSE turbo equalizers," IEEE Comm. Letters, vol. 5, no. 7, pp. 310-312, 2001.

[15] M. Tüchler and J. Hagenauer, "Linear time and frequency domain turbo equalization," IEEE Veh. Technol. Conf. (VTC 2002), vol. 4, pp. 27732777, 2002.

[16] S. Moshavi, E. Kanterakis, and D. Schilling, "Multistage linear receivers for DS-CDMA systems," Int. J. Wireless Inform. Networks, vol. 3, no. 1, pp 1-17, Jan. 1996.

[17] C. Boulanger, L. Ouvry, and M. des Noes, "Multistage Linear DSCDMA Receivers," IEEE Int. Symp. on Spread Spectrum Techniques and Applications (ISSSTA 98), vol. 2, pp 663-667, Sept. 1998.

[18] J. Goldstein and I. Reed, "Reduced-Rank Adaptive Filtering," IEEE-T$S P$, vol. 45, no. 2, pp 492-496, Feb. 1997.

[19] G. Dietl, I. Groh, and W. Utschick, "Low-Complexity MMSE Receivers Based on Weighted Matrix Polynomials in Frequency-Selective MIMO Systems," Int. Symp. on Signal Proc. and Its Applications (ISSPA 2005), vol. 2, pp 699-702, Aug. 2002.

[20] G. Golub and C. Van Loan, "Matrix Computations," John Hopkins Univ. Press, 1989.

[21] M. Botsch, G. Dietl, and W. Utschick, "Iterative Multi-User Detection Using Reduced-Complexity Equalization," Proc. Int. Symp. Turbo Codes, vol. 2, pp 699-702, April 2006.

[22] R. Müller and S. Verdù, "Design and Analysis of Low-Complexity Interference Mitigation on Vector Channels," IEEE Journal on Selected Areas in Comm., vol. 19, no. 8, pp 1429-1441, Aug. 2001.

[23] N. Le Josse, C. Laot, and K. Amis, "Efficient series expansion for matrix inversion with application to MMSE equalization," IEEE Comm. letters, vol. 12 , no. 1, pp. 35-37, Jan. 2008.

[24] Z. Lei and T. Lim, "Simplified Polynomial-expansion Linear Detectors for DS-CDMA Receivers," IEEE Elec. Letters, vol. 34, no. 16, pp 15611563, 1998

[25] M. Mozaffaripour and R. Tafazolli, "Suboptimum Search Algorithm in Conjunction With Polynomial Expanded Multiuser Detection for Uplink," Wireless Personnal Comm., Kluwer Academic Publishers, vol. 24, no. 1, pp 1-9, 2003.

[26] C. Laot, R. Le Bidan, and D. Leroux, "Low complexity linear turbo equalization: A possible solution for EDGE," IEEE Trans. Wireless. Comm., vol. 4, no. 3, pp 965-974, May 2005.

[27] M. Witzke, S. Bäro, F. Schreckenbackand, and J. Hagenauer, "Iterative detection of MIMO signals with linear detectors," Proc. Asilomar Conf. Signals, Systems, Computers, Pacific Grove, CA, Nov. 2002.

[28] K. Amis, N. Le Josse, and C. Laot, "Efficient Frequency-Domain MMSE turbo equalization derivation and performance comparison with the Time-Domain counterpart," Int. Conf. on Wireless and Mobile Comm. (ICWMC 2007), March 2007.

[29] J. Pan, C. Soh, and E. Gunawan, "Simplified Iterative MMSE Multiuser Detector Using Polynomial-Expansion Approximation," Wireless Personnal Comm., Kluwer Academic Publishers, vol. 24, no. 1, pp 1-9, 2003. 